\title{
Better Together: Joint Reasoning for Non-rigid 3D Reconstruction with Specularities and Shading
}

\author{
Qi Liu-Yin ${ }^{1}$ \\ Qi.Liu@cs.ucl.ac.uk \\ Rui Yu ${ }^{1}$ \\ R.Yu@cs.ucl.ac.uk
}

Andrew Fitzgibbon²

awf@microsoft.com

\author{
${ }^{1}$ University College London \\ London, UK \\ ${ }^{2}$ Microsoft Research Cambridge \\ Cambridge, UK
}

\section{Lourdes Agapito ${ }^{1}$ \\ L.Agapito@cs.ucl.ac.uk \\ Chris Russell ${ }^{1}$ \\ crussell@turing.ac.uk}

In this paper, we demonstrate the use of shapefrom-shading ( $\mathrm{S} f \mathrm{~s}$ ) to improve both the quality and the robustness of $3 \mathrm{D}$ reconstruction of dynamic objects captured by a single camera. Unlike previous approaches that made use of $\mathrm{s} f \mathrm{~S}$ as a post-processing step, we offer a principled integrated approach that solves dynamic object tracking and reconstruction and $\mathrm{s} f \mathrm{~S}$ as a single unified cost function. Moving beyond Lambertian $\mathrm{s} f \mathrm{~s}$, we propose a general approach that models both specularities and shading while simultaneously tracking and reconstructing general dynamic objects. Solving these problems jointly prevents the kinds of tracking failures which can not be recovered from by pipeline approaches.
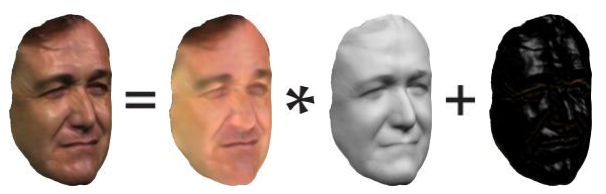

Figure 1: The reflected intensity is the product of albedo and diffuse shading plus specularities.

Our proposed approach is an online templatebased method that captures both the 3D geometry and the reflectance properties (Figure 1) of the non-rigid object. Our main novelty is the photometric error data term of the energy cost that is minimized for each new frame. It models the photometric error as follows

$E_{D}=\sum_{i \in \mathcal{V}}\left\|\mathbf{I}\left(\pi\left(\mathbf{R}\left(\mathbf{s}_{i}\right)+\mathbf{t}\right)\right)-\widehat{\boldsymbol{\rho}}_{i} \mathbf{l} \cdot Y\left(\mathbf{R}\left(\mathbf{n}_{i}(\boldsymbol{S})\right)\right)-\boldsymbol{\beta}_{i}\right\|_{\varepsilon}$

For each vertex, it penalizes the difference between its projected and its estimated intensities as a function of albedo $\widehat{\boldsymbol{\rho}}$, diffuse shading $\mathbf{l} \cdot Y(\cdot)$ and specular highlights $\boldsymbol{\beta}$.

We tested our method on synthetically rendered sequences, using the results from [1], and on real sequences. We compare against [2] and show state-of-the-art results both qualitatively (Figure 2) and quantitatively (Table 1).

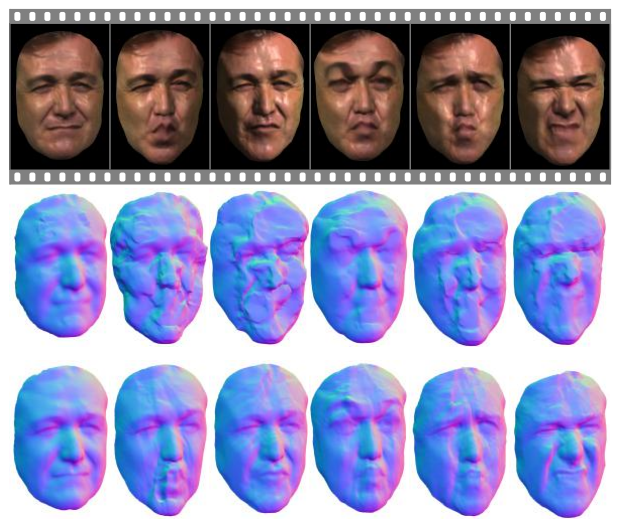

Figure 2: From top to bottom: synthetic input sequence, results from Yu et al., and our results.

\begin{tabular}{|c||c|c|c|c|}
\hline & LF & SF & LC & SC \\
\hline Yu et al. $[2]$ & 7.29 & 7.93 & 9.18 & 9.28 \\
\hline Ours & 2.73 & 2.89 & 3.42 & 3.84 \\
\hline
\end{tabular}

Table 1: Comparison of RMS error (in mm.) with Yu et al. on 4 different synthetic sequences.

[1] Levi Valgaerts et al. Lightweight binocular facial performance capture under uncontrolled lighting. SIGGRAPH Asia, 2012.

[2] Rui Yu et al. Direct, dense, and deformable: Nonrigid $3 \mathrm{~d}$ reconstruction from $\mathrm{rgb}$ video. ICCV, 2015 . 